\title{
Rough Fresnel zone plates over metallic surfaces
}

\author{
Francisco Javier Salgado-Remacha, ${ }^{\star}$ Luis Miguel Sanchez-Brea, \\ Francisco Javier Alvarez-Rios, and Eusebio Bernabeu \\ Applied Optics Complutense Group, Optics Department, Universidad Complutense de Madrid, \\ Facultad de Ciencias Físicas, Ciudad Universitaria s.n., 28040 Madrid, Spain \\ ${ }^{*}$ Corresponding author: fjsalgado@fis.ucm.es
}

Received 29 October 2009; revised 4 February 2010; accepted 8 March 2010; posted 9 March 2010 (Doc. ID 119206); published 24 March 2010

\begin{abstract}
We analyze the focusing properties of Fresnel zone plates fabricated over steel tapes using laser ablation. Our intention is to implement the use of micro-optical elements when the use of conventional chromeglass elements is not indicated. Because of the manufacture process, the surface presents a certain anisotropic roughness, which reduces the focusing properties. First, we develop numerical simulations by means of the Rayleigh-Sommerfeld approach, showing how roughness in both levels of the Fresnel zone plate affects the focalization of the lens. We also manufacture Fresnel zone plates over steel tape, and perform experimental verification that corroborates the numerical results. (C) 2010 Optical Society of America

OCIS codes: $\quad 050.1965,240.3990,240.5770$.
\end{abstract}

\section{Introduction}

Micro- and nano-optics have experienced increasing development in recent years. Micro-optical elements are successfully used in several applications, such as optical metrology, adaptive optics, and optical trapping, [1,2]. Fresnel zone plates (FZPs) are one of the most used micro-optical elements because they allow the focusing of a light beam with advantages over conventional lenses: they can be used, for example, over spectral regions where refractive lenses are useless (as UV), or in applications where their lower weight (in comparison with refractive lenses) suggests their use. The typical flat arrangement is well suited for the integration of diffractive lenses within a micro-optics setup [3,4]. On the other hand, FZPs present some disadvantages, such as their worse optical behavior. For example, a FZP presents a principal focus, and a collection of higher-order foci. The appearance of multifoci implies the reduction of optical efficiency, and a worse concentration capability in the principal focus. With regard to a binary-

0003-6935/10/101750-07\$15.00/0

(C) 2010 Optical Society of America amplitude FZP, a modulation in amplitude implies loss of irradiance at the observation plane.

Commonly, chrome-over-glass masks, dielectric materials (glass, plastics), or semiconductors (silica) are used as substrates. Photolithographic techniques are commonly used for the manufacture of binary diffractive elements, with a very high accuracy after decades of developing chrome-over-glass masks for the semiconductor industry. Nevertheless, there exist applications where the environmental conditions are extreme due to vibrations, impacts, or thermal variations. In such cases, it is necessary to fabricate micro-optical elements over more robust substrates, such as steel tapes. An easy way to engrave over metallic tapes is to use laser ablation, a technique for manufacturing micro-optical elements over a high number of materials, such as metals [5], ceramics [6] or glasses [7]. The technique consists of removing material by means of focusing onto the sample a high-power light beam, with several processes involved during the process [8].

The optical behavior of these elements is not optimal, due to the surface roughness of steel. Also, the roughness topography is anisotropic, due to the fabrication process of the steel tape. For simple structures, such as diffraction gratings, it is possible to 
develop theoretical models that allow us to predict their optical behavior [9-11]. For more complex devices, it is not always possible to carry out an analytical treatment. Thus, other design methods are required, such as numerical simulations and experimental analysis.

In this work, we analyze the optical properties of rough FZPs (RFZPs) manufactured on steel tape using laser ablation. A statistical model is used to describe the roughness parameters of the anisotropic surface. We center our analysis in RFZP with two different levels of roughness (on one hand, the roughness of the steel substrate and, on the other hand, the roughness produced by the laser ablation process) working in a reflection configuration. Moreover, anisotropy due to steel fabrication processes has been also included. We will analyze the focusing properties of RFZPs in terms of the statistical properties of roughness. A generalized analytical method, including two different roughness levels and anisotropy, seems to be unattainable. The results obtained for the RFZPs can be extended to any binary diffractive element engraved on a steel tape. For the numerical analysis, we have used the scalar theory for light propagation, in particular Rayleigh-Sommerfeld approach. Referring to roughness descriptions, we assume a Gaussian distribution of surface heights (based on experimental data). Roughness should also be higher than wavelength.

In Section 2, we present the mathematical expressions needed to describe the RFZP. In Section 3, we numerically characterize the optical behavior of the RFZP by means of the Rayleigh-Sommerfeld diffraction integral, using a fast-Fourier-transform-based direct integration (FFT-DI) method [12]. In Section 4, we explain the process used to fabricate the lenses and the typical topography obtained with this process, and we perform also an experimental analysis of manufactured lenses. Conclusions are given in Section $\underline{5}$.

\section{Rough Fresnel Zone Plates}

A FZP consists of alternately opaque and transparent rings (in the transmission case) or reflective and nonreflective rings (in the reflective case). These elements have been known for more than 100 years [13-15], and they can be manufactured by laser ablation. It is defined by

$$
L(x, y)= \begin{cases}1 & \text { if } \sqrt{m \lambda f} \leq x^{2}+y^{2}<\sqrt{(m+1) \lambda f} \\ 0 & \text { if } \sqrt{(m-1) \lambda f} \leq x^{2}+y^{2}<\sqrt{m \lambda f}\end{cases}
$$

where $\lambda$ is the wavelength, $f$ is the focal length of the FZP, and $m$ is an integer $m=1,2,3 \ldots$. With this definition, the central ring is opaque, and a FZP is called then, an "even" or a "negative" FZP. In Fig. 1 (a), we show an example of a negative FZP designed following Eq. (1), for a focal length $f=450 \mathrm{~mm}$.
Since the RFZP is engraved on a steel tape, we need to know the topographical properties of roughness. Let us assume that steel samples present stochastic topography $h_{1}(x, y)$ [16]. Let us assume that the statistical height distribution of the topography, $p(h)$, presents a Gaussian distribution. Considering that the mean height of the topography is null, $\langle h\rangle_{s}=$ $\int_{-\infty}^{\infty} h p(h) \mathrm{d} h=0$, where \langle\rangle$_{s}$ denotes spatial averaging, the standard deviation of heights is given by

$$
\sigma=\sqrt{\left\langle h^{2}\right\rangle_{s}}
$$

Another important parameter to characterize the surface roughness is the correlation distance, which can be determined from the autocorrelation function of $h(x, y)$, defined by $C(R)=\langle h(r) h(r+R)\rangle_{s} / \sigma^{2}$, where $r$ is the spatial coordinate. The surface correlation function is normally assumed as Gaussian

$$
C(R)=\exp \left(-\frac{R^{2}}{T^{2}}\right),
$$

where $T$ is the correlation length, being the distance over which the correlation function falls by $1 / e$, and indicates the rate of change of surface height along the surface. Because of the fabrication process, the surface is not normally isotropic. Therefore, we need to define the correlation length for each direction, $T_{x}$ and $T_{y}$.

The ablation process produces a local change in the topography. Moreover, a superficial ablation of the material produces a change in roughness, and a slight variation in the mean depth, as we will see in Section 4. Under this condition, the ablation process modifies the original topography $h_{1}(x, y)$ to a new topography $h_{2}(x, y)$ with different statistical properties. Then, we will describe the statistical properties of the steel surface by $T_{1 x}, T_{1 y}$, and $\sigma_{1}$, and those of the ablationed zone by $T_{2 x}, T_{2 y}$, and $\sigma_{2}$.

Finally, the reflectance of the RFZP is

$$
\begin{aligned}
r(x, y)= & L(x, y) \exp \left[-2 i k h_{1}(x, y)\right] \\
& +[1-L(x, y)] \exp \left[-2 i k h_{2}(x, y)\right] .
\end{aligned}
$$

Equation (3) allows us to generalize the description of any binary diffractive element over a steel tape, not only to FZPs. In this general case, $L(x, y)$ is the expression for the shape of the diffractive element, that is, the nonablationed area.

To determine the field after the RFZP, let us consider a plane wave $U_{0}(\xi, \eta)=A_{0}$ illuminating the diffractive element $L(x, y)$. The near field can be determined using the Fresnel integral:

$$
U(x, y, z)=\frac{A_{0} e^{i k z}}{i \lambda z} \iint r(\xi, \eta) e^{i \frac{k}{2 z}\left[(x-\xi)^{2}+(y-\eta)^{2}\right]} \mathrm{d} \xi \mathrm{d} \eta,
$$

where $z$ is the distance between the diffractive element and the observation plane, $\lambda$ is the wavelength, and $k=2 \pi / \lambda$. 


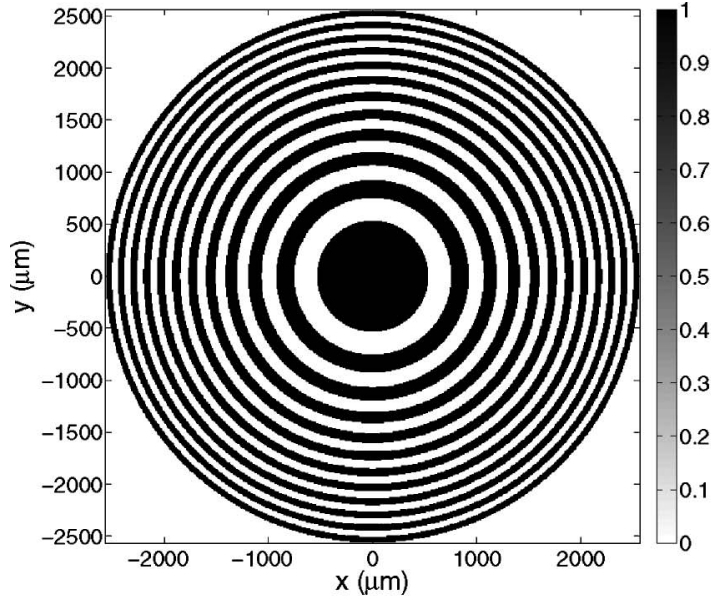

(a)

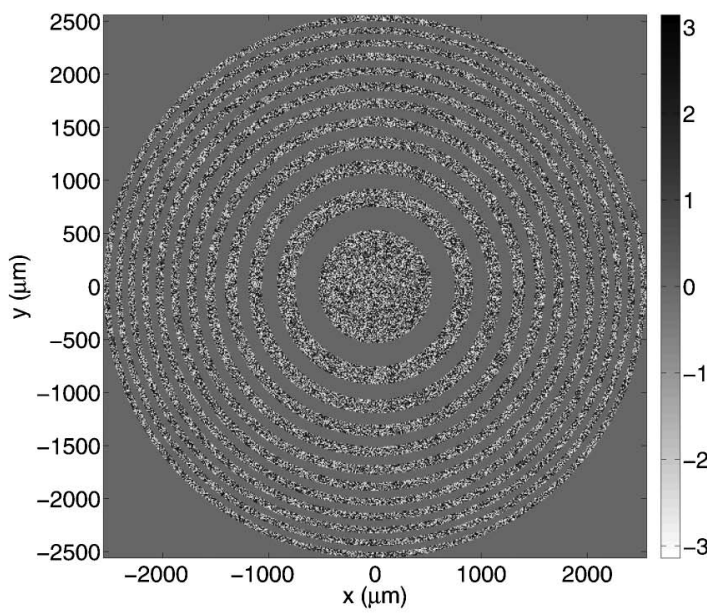

(b)

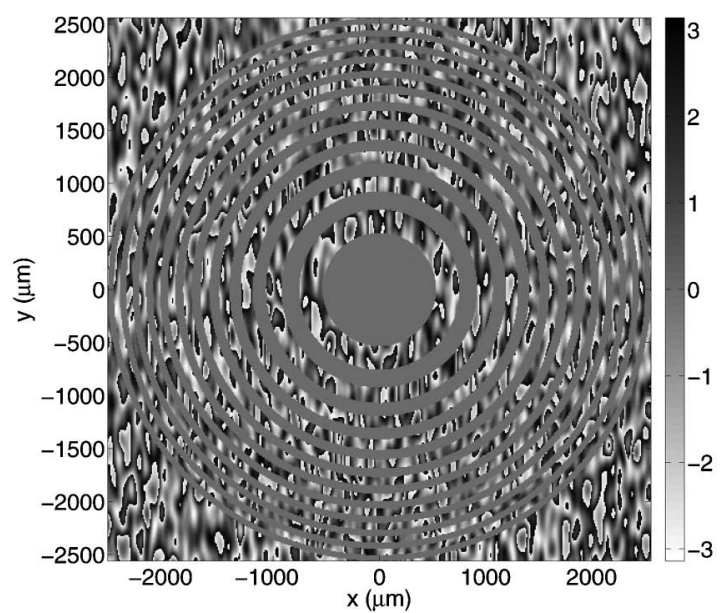

(c)

Fig. 1. Images of the Fresnel zone plate used in the simulations. The FZP presents a focal length of $f=450 \mathrm{~mm}$. (a) Ideal FZP. (b) Simulation of a RFZP engraved by laser ablation (data from Table 1 : $\sigma_{2}=0.27 \mu \mathrm{m}, T_{2 x}=4.2 \mu \mathrm{m}, T_{2 y}=4.8 \mu \mathrm{m}$ ) over a flat surface, $\sigma_{1}=0 \mu \mathrm{m}$. (c) Simulation of a RFZP engraved by laser ablation over a real surface with low roughness level (data from Table 1: $\sigma_{1}=0.05 \mu \mathrm{m}$, $\left.T_{1 x}=50.23 \mu \mathrm{m}, T_{1 y}=164.38 \mu \mathrm{m}\right)$ but with $\sigma_{2}=0 \mu \mathrm{m}$.

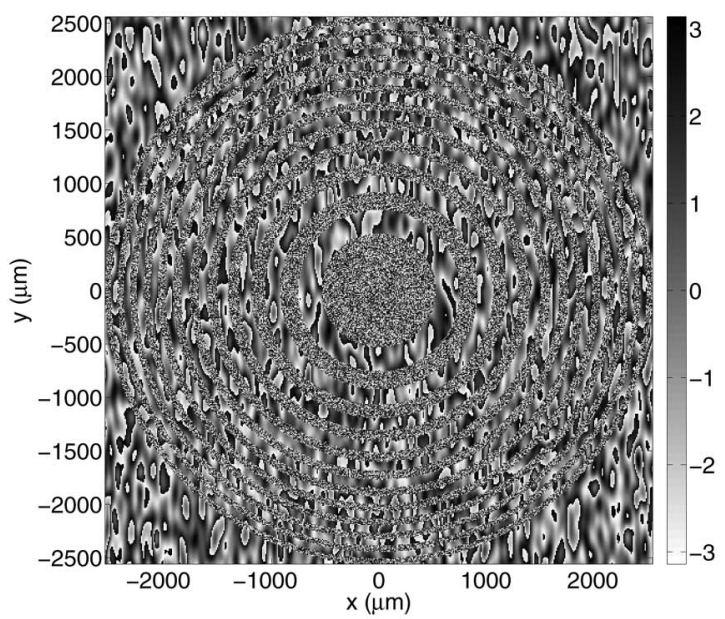

(a)

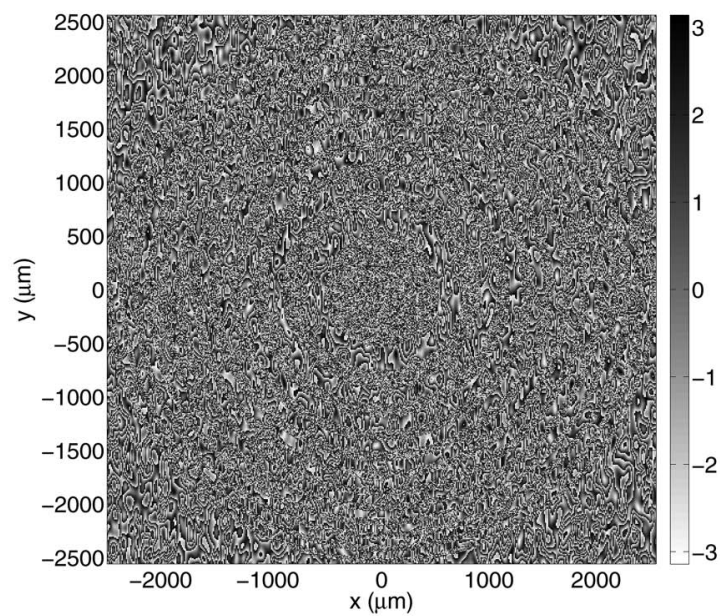

(b)

Fig. 2. (a) Simulation of a RFZP engraved by laser ablation over a real surface with low roughness level (data from Table 1: $\sigma_{1}=0.05 \mu \mathrm{m}$, $T_{1 x}=50.23 \mu \mathrm{m}, T_{1 y}=164.38 \mu \mathrm{m}$ ). (b) Simulation of a RFZP engraved by laser ablation over a real surface with high roughness level (data from Table $\left.\underline{1}: \sigma_{1}=0.15 \mu \mathrm{m}, T_{1 x}=11.60 \mu \mathrm{m}, T_{1 y}=119.20 \mu \mathrm{m}\right)$. 
An analytical development of Eq. (4), taking into account a rough and anisotropic topology, is hard and tedious. On the other hand, numerical methods lead us to analyze the optical behavior of any rough binary diffractive element, regardless of its forms. In Section 3, we explain how to determine the optical field diffracted by a RZFP.

\section{Numerical Simulations of Rough Diffractive Lenses}

To solve numerically the Rayleigh-Sommerfeld diffraction integral we have used a FFT-DI method [12]. The approach is accurate when the features of the mask (the FZP) are larger than the wavelength. In addition, we need to simulate surfaces with stochastic topology. We make use of software based on Ref. [17] to generate virtual topologies that present a selected correlation length and a standard deviation, assuming that the spatial distribution fol-
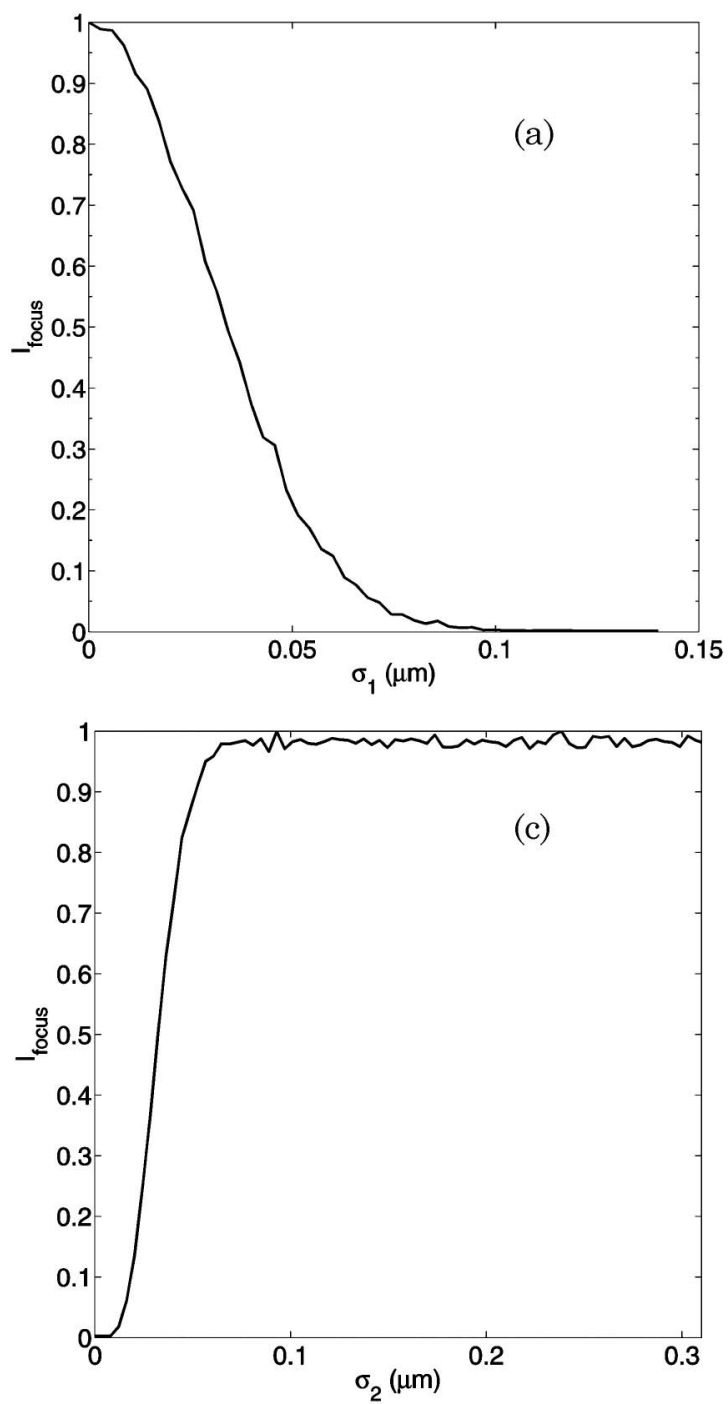

lows a Gaussian distribution. Rough topology is included as phase variations over the RFZP.

For the simulations, we consider the experimental conditions detailed in Section 4. The light source is a plane wave with wavelength $\bar{\lambda}=632.8 \mathrm{~nm}$, which illuminates the rough RFZP in normal incidence. Here we have used the FZP from Fig. 1(a) $(f=450 \mathrm{~mm}$, $512 \times 512$ pixel number) as a guide for our simulations. The roughness values are suggested by experimental results, collected in Table 1 and explained in detail in Section 4. When the FZP is engraved on a flat surface (without roughness), the result is similar to that shown in Fig. 1(b), where the rough surface simulates the ablation process. On the other hand, a flat FZP engraved over a rough surface is shown in Fig. 1(c). Additionally, two examples of RFZPs with $\sigma_{2}$ from Table 1 and two different values for $\sigma_{1}$ (that means, with different values of roughness
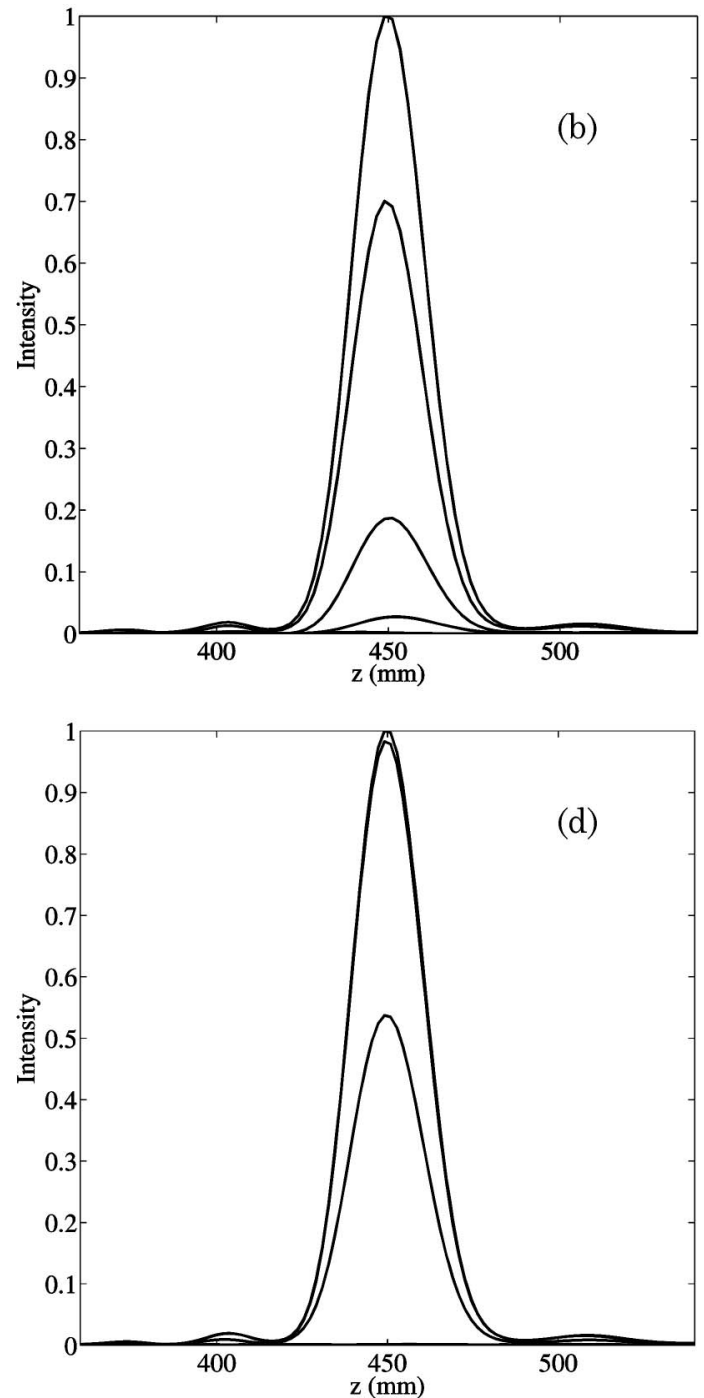

Fig. 3. Dependence of the focusing capability with roughness. (a) Maximum of intensity at the focal plane for different values of $\sigma_{1}$, with $\sigma_{2}=4.5 \mu \mathrm{m}$. (b) Irradiance profiles along the $z$ axis for different values of $\sigma_{1}: 0$ (highest), 0.01, 0.03, 0.05, and $0.07 \mu \mathrm{m}$ and the same value for $\sigma_{2}=4.5 \mu \mathrm{m}$, normalized to the maximum. (c) Maximum of intensity at the focal plane for different values of $\sigma_{2}$, with $\sigma_{2}=0.05 \mu \mathrm{m}$. (d) Irradiance profiles along the $z$ axis for different values of $\sigma_{2}: 0$ (lowest), $0.03,0.06$, and 0.1 , and the same value for $\sigma_{1}=0.05 \mu \mathrm{m}$, normalized to the maximum. 


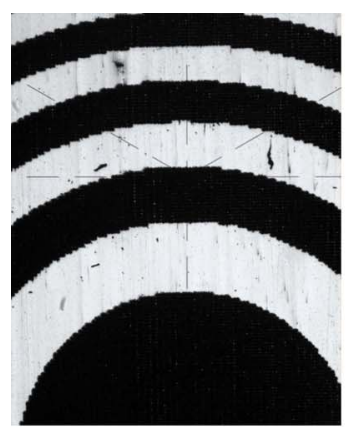

(a)

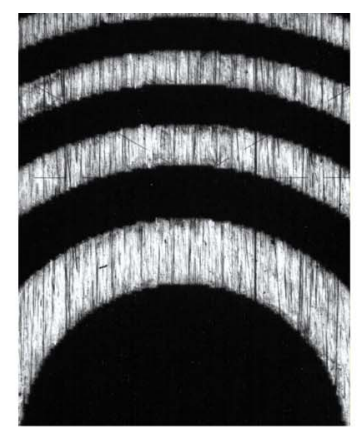

(b)

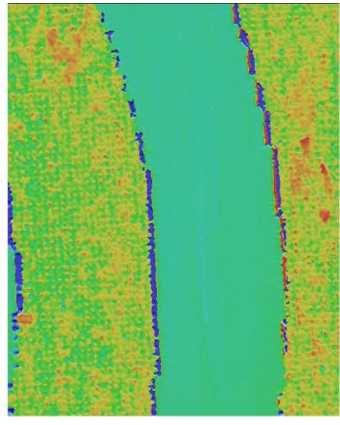

(c)

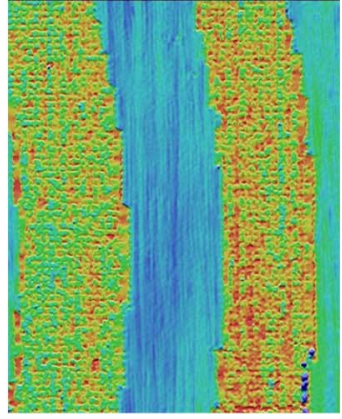

(d)

Fig. 4. (Color online) (a) and (b) Optical microscopy image of a RFZP for low and high roughness steel substrates respectively. (c) and (d) Confocal microscopy image of a RFZP for low and high roughness steel substrates, respectively.

over the samples without the ablation process) are shown in Figs. 2(a) and 2(b), emulating the real RFZP manufactured. These masks will be used in our simulations.

To understand the importance of the substrate roughness, we first analyze the dependence of the focusing capability of the RFZP in terms of the standard deviation of the substrate, $\sigma_{1}$. In Fig. 3(a). the maximum intensity at the focal plane is shown for FZPs with a lens similar to the lenses shown in Fig. 1, but for different values of $\sigma_{1}$, normalized to the maximum (obtained with $\sigma_{1}=0$ ). The intensity at the focus decays strongly as $\sigma_{1}$ increases. The intensity profile along the $z$ axis (propagation axis) for five different values of $\sigma_{1}$ is shown in Fig. 3(b), normalized again to the maximum. We can appreciate how the focusing capability decreases as $\sigma_{1}$ increases. It is also worth noting that the depth of focus remains unchanged, despite the variation in $\sigma_{1}$. In Figs. 3(c) and $3(\mathrm{~d})$, we show the same results, but varying $\sigma_{2}$ [corresponding to Fig. 1(c)]. Also, the depth of focus is independent on $\sigma_{2}$. For this case, there exists a superior limit for the focusing capability. By means of laser ablation, it is possible to reach this limit, but not to go beyond it. This fact lead us to center our discussion on the effect of $\sigma_{1}$, as the appropriate parameter to control the optical behavior of the RFZP.

\section{Experimental Results}

The FZP of Fig. 1 has been engraved over two different samples of steel using laser ablation. We have used an ablation laser system composed of a two-axis Newport platform and a $1064 \mathrm{~nm} Q$-switched laser (Navigator I-Spectra-Physics Laser) with $10 \mathrm{~W}$ of

Table 1. Roughness Values Obtained by Confocal Microscopy from the Two Manufactured Rough Fresnel Zone Plates

\begin{tabular}{cccc}
\hline & $T_{x}$ & $T_{y}$ & $\sigma$ \\
\hline $\begin{array}{c}\text { Substrate with low } \\
\text { roughness level }\end{array}$ & $50.23 \mu \mathrm{m}$ & $164.38 \mu \mathrm{m}$ & $0.06 \mu \mathrm{m}$ \\
$\begin{array}{c}\text { Substrate with high } \\
\text { roughness level }\end{array}$ & $11.60 \mu \mathrm{m}$ & $119.20 \mu \mathrm{m}$ & $0.16 \mu \mathrm{m}$ \\
$\begin{array}{c}\text { Substrate after } \\
\text { ablation process }\end{array}$ & $4.20 \mu \mathrm{m}$ & $4.80 \mu \mathrm{m}$ & $0.26 \mu \mathrm{m}$ \\
\hline
\end{tabular}

peak power. To minimize the spot size, we also use a third harmonic generator, thus the final operating wavelength is $355 \mathrm{~nm}$, with $2 \mathrm{~W}$ of peak power. With this configuration, the Gaussian size of the spot is about $3 \mu \mathrm{m}$. The system is arranged to work as a plotter, so we can manufacture a mask pixel by pixel. The manufactured RFZPs are shown in Fig. 4(a) (with a low-roughness-level steel sample) and Fig. 4(b) (with a high-roughness-level steel sample).

We have obtained the surface topography of the samples [Figs. 4(c) and 4(d)] from images obtained using a confocal microscope $(\mathrm{Pl} \mu$ by Sensofar, Spain). The roughness parameters of the RFZP measured from the images have been collected in Table 1 . The correlation length depends on the direction, due to the fabrication process of the steel tapes. Therefore, we have used $T_{x}$ and $T_{y}$ to simulate the correlation length in the $x$ and $y$ directions.

To perform the experimental verification of the results obtained from the simulations, we have used the setup shown in Fig. 5. The beam produced by a fiber-pigtailed laser diode (FPLD), with $\lambda=$ $632.8 \mathrm{~nm}$, impinges over the RFZP in normal incidence. The use of a fiber-pigtailed diode ensures a filtered and Gaussian-like beam, and, with a collimation lens (CL), we obtain a quasi-plane wavefront to illuminate the RFZP. To obtain a perpendicular illumination, we use a 50/50 beam splitter (BS) placed at $45^{\circ}$ with the beam propagation direction. The light reflected by the RFPZ is redirected through the BS to

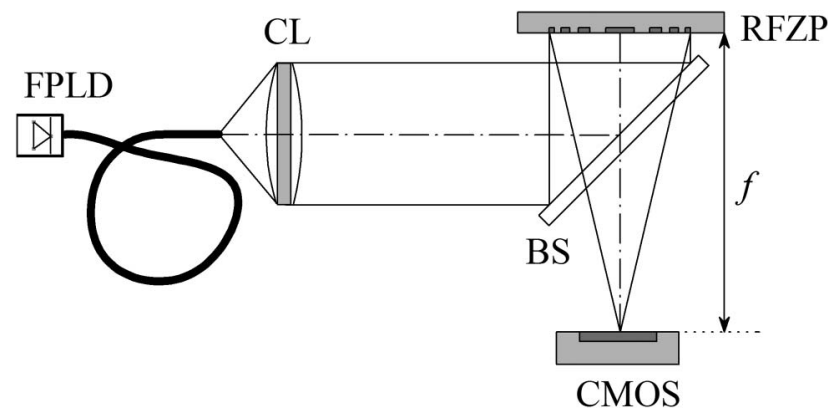

Fig. 5. Experimental setup. FPLD is a fiber pigtailed laser diode, CL is a collimation lens, BS is a beam splitter, and $f$ is the focal length of the RFZP. 


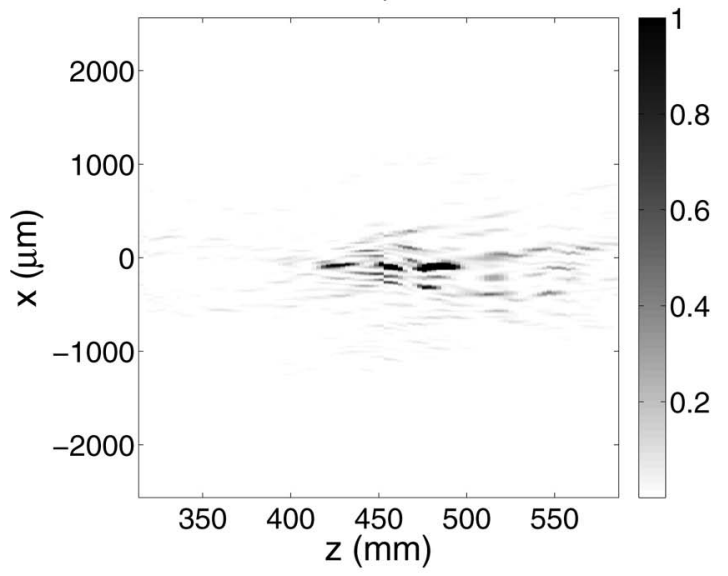

(a)

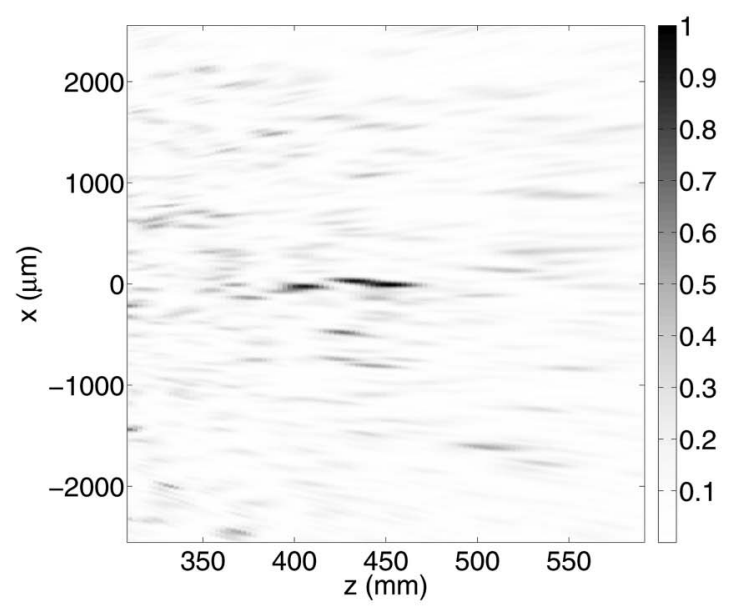

(c)

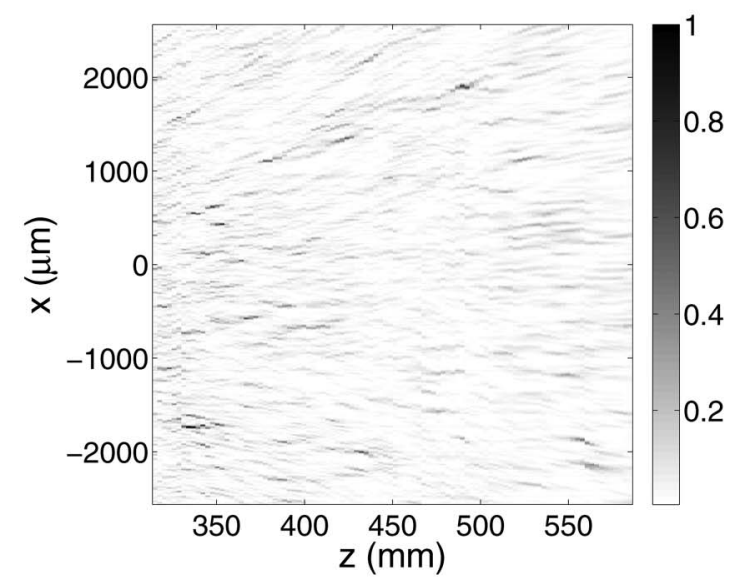

(b)

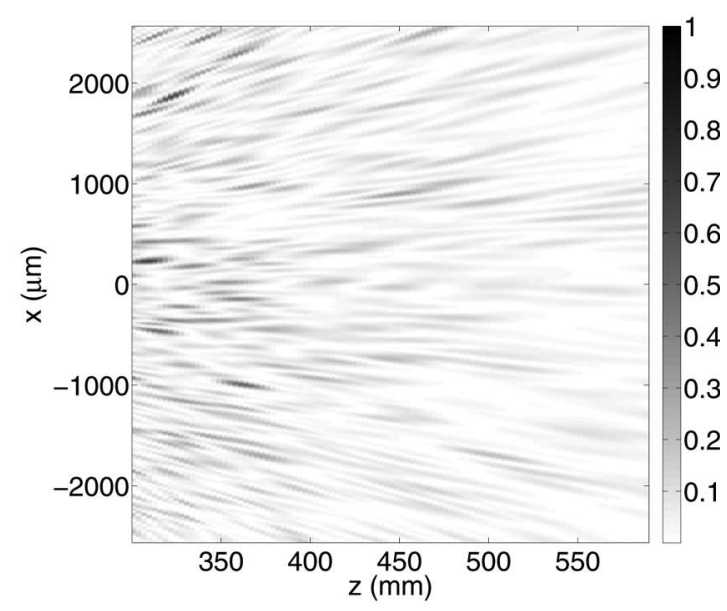

(d)

Fig. 6. (a) and (b) Transversal view of the propagation of light with RFZP from Figs. 4(a) and 4(b), respectively (experimental data). (c) and (d) Simulations with the Rayleigh-Sommerfeld approach of propagation of light from Figs. $\underline{4(\mathrm{a})}$ and $\underline{4(\mathrm{~b})}$ respectively. All the intensities are normalized to the unit.

a CMOS (UI-1220-M by U-Eye, Germany), with a pixel pitch of $6 \mu \mathrm{m}$. The camera is mounted over a motorized linear stage, so it can travel along the propagation axis and images can be acquired at any desired plane. Under these conditions, we have obtained the intensity distribution along the propagation axis around the focus of the RFZPs. In Fig. 6 , we show a transversal view of the propagation of the light using a RFZP with low roughness level [Fig. 6(a)] and with high roughness level [Fig. 6(b)], obtained experimentally with the described setup. The substrates in which lenses were engraved can induce optical aberrations, because of the overall lack of flatness of the surface. For comparison, we show the Rayleigh-Sommerfeld simulations for the same cases in Figs. 6(c) and 6(d), respectively. The simulated lenses are not exactly equal to the fabricated lenses, but they have the same statistical parameters. Light concentration clearly appears for low roughness of the steel substrate, whereas, for high roughness, the RFZP does not present focusing capability, corroborating the predictions shown in Fig. $\underline{3}$.
The simulations shown in Figs. 6(c) and 6(d) also show a concentration of light around the focus for the low-roughness-level FZP, and the absence of light concentration for a high roughness level. A complete coincidence between experimental and simulated results is not expected, since we do not use exactly the same topography for the simulation and the experiment, but they have the same statistical properties.

\section{Conclusions}

In the present work, we have fabricated FZPs over steel using an ablation laser. The effect of surface roughness of the steel tape over the focusing properties of the FZP has been analyzed using numerical simulations in terms of the standard deviation in heights. We have shown that the focusing properties of the RFZP depend strongly on roughness of the steel sample, which presents an anisotropic topology. The intensity at the focus decays with the standard deviation of the roughness of the substrate. At the same time, we have shown that roughness from ablationed zones is not an important parameter, 
whenever we reach a certain level. Experiments with RFZPs corroborate numerical predictions, showing that the surface quality of the sample is the most important parameter in order to improve the optical behavior of the RFZP.

The authors are grateful to Francisco José Torcal Milla for his help. This work has been partially supported by project CCG08-UCM/DPI-3952 of Dirección General de Universidades e Investigación de la Consejería de Educación de la Comunidad de Madrid y Universidad Complutense de Madrid and project DPI2008-02391 of Ministerio de Ciencia e Innovación. Salgado-Remacha acknowledges the economical support of the Ministerio de Ciencia e Innovación.

\section{References}

1. J. Turunen and F. Wyrowski, Diffractive Optics for Industrial and Commercial Applications (Wiley-VCH, 1998).

2. H. P. Herzig, Micro-Optics. Elements, Systems and Applications (Taylor \& Francis, 1997).

3. F. J. González, J. Alda, B. Illic, and G. Boreman, "Infrared antennas coupled to lithographic Fresnel zone plate lenses," Appl. Opt. 43, 6067-6073 (2004).

4. J. Alda, J. M. Rico-García, J. M. López-Alonso, B. Lail, and G. Boreman, "Design of Fresnel lenses and binary-staircase kinoforms of low value of the aperture number," Opt. Commun. 260, 454-461 (2006).

5. X. Zhu, D. M. Villeneuve, A. Yu. Naumov, S. Nikumb, and P. B. Corkum, "Experimental study of drilling sub-10 $\mu \mathrm{m}$ holes in thin metal foils with femtosecond laser pulses," Appl. Surf. Sci. 152, 138-148 (1999).
6. M. F. Modest, "Transient elastic and viscoelastic thermal stresses during laser drilling of ceramics," J. Heat Transfer 120, 892-898 (1998).

7. J. Krüger and W. Kautek, "Femtosecond-pulse visible laser processing of transparent materials," Appl. Surf. Sci. 96-98, 430-438 (1996).

8. J. Noack and A. Vogel, "Laser-induced plasma formation in water at nanosecond to femtosecond time scales: calculation of thresholds, absorption coefficients, and energy density," IEEE J. Quantum Electron. 35, 1156-1167 (1999).

9. F. J. Torcal-Milla, L. M. Sanchez-Brea, and E. Bernabeu, "Talbot effect in rough reflection gratings," Appl. Opt. 46, 3668-3673 (2007).

10. L. M. Sanchez-Brea, F. J. Torcal-Milla, and E. Bernabeu, "Talbot effect in metallic gratings under Gaussian illumination," Opt. Commun. 278, 23-27 (2007).

11. F. J. Torcal-Milla, L. M. Sanchez-Brea, and E. Bernabeu, "Double grating systems with one steel tape grating," Opt. Commun. 281, 5647-5652 (2008).

12. F. Shen and A. Wang, "Fast-Fourier-transform based numerical integration method for the Rayleigh-Sommerfeld diffraction formula," Appl. Opt. 45, 1102-1110 (2006).

13. R. W. Wood, "Phase-reversal zone plates, and diffractiontelescopes," Philos. Mag. 45, 511-522 (1898).

14. A. V. Baez, "Fresnel zone plate for optical image formation using extreme ultraviolet and soft $\mathrm{x}$ radiation," J. Opt. Soc. Am. 51, 405-412 (1961).

15. J. Kirz, "Phase zone plates for $\mathrm{x}$ rays and the extreme uv," J. Opt. Soc. Am. 64, 301-309 (1974).

16. J. A. Ogilvy, Theory of Wave Scattering From Random Rough Surfaces (Taylor \& Francis, 1991).

17. P. Beckmann and A. Spizzichino, The Scattering of Electromagnetic Waves form Rough Surfaces (Artech House, 1987), Chap. 5. 